\title{
FPAA Based Design of Assistive Listening Device for Hearing Disorders People

\author{
${ }^{1}$ Department of Electronics \& Telecommunication, Kamalnayan Bajaj Institute of Engineering \& Technology, \\ Baramati \\ ${ }^{2}$ Department of Electronics Engineering, Shri Ramdeobaba College of Engineering \& Management, Nagpur, India \\ Email:landeeed@yahoo.com,ssbalpande@gmail.com
} \\ ${ }^{1}$ Dr. Sudhir B. Lande, ${ }^{2}$ Suresh S. Balpande
}

Received: $20^{\text {th }}$ September 2018, Accepted: $11^{\text {th }}$ October 2018, Published: $31^{\text {st }}$ October 2018

\begin{abstract}
An assistive listening device (ALD) beyond hearing aids can assist individuals with hearing loss. The traditional aid is similar to a radio, which can be adjusted for volume. The hearing loss is not just a technical loss of volume. Hearing disorder looses sensitivity and reduces tolerance to certain sounds while diminishing sensitivity to others. For instance, technology can differentiate between speeches and noise allowing one while filtering out the other. Customizable ALD that amplifies sound non-uniformly over the hearing spectrum according to the client's audiogram to compensate for frequency dependent hearing deficiencies is proposed. An analog system has been developed using ANADIGM ${ }^{\mathrm{TM}}$ synthesis tool for FPAA (Field Programmable Analog Arrays) device. An innovative technique that can be used to screen hearing impaired people to enable the easier fitting of listening device has been investigated. This proposed technique is easy to handle and can be used as a clinical or research tool. This work also covers the development of mold and ear fitment using biocompatible PDMS $\left(\left(\mathrm{C}_{2} \mathrm{H}_{6} \mathrm{OSi}\right)\right.$ n-Polydimethylsiloxane $)$ polymer .
\end{abstract}

\section{Keywords}

Hearing Listening Devices, FPAA, PDMS Based Fitment

\section{Introduction}

Life expectancy is continuously increasing due to advancement in medical field, but hearing loss due to aging has been one of the most common problem of the mass ${ }^{1,2,3}$. Lots of confusions may arise due to hearing loss which motivates us to design assistive device .A variety of hearing assistive technologies are developed by researchers and recommended for whose hearing loss is moderate ${ }^{4}$. The era of low power, high-complexity electronics supports the implementation of ALD. The main objective is to provide the user with a listening experience through the device that resembles natural hearing of the sound information ${ }^{5,6}$. The ALD is a miniaturized device mounted in or around the ear to improve the hearing quality. The basic components comprises of speaker, microphone, FPAA based analog subsystem, interface unit and a battery. There are four styles of ALD like behind the ear (BTE), in the ear (ITE), in the canal (ITC), and completely in the canal (CIC). There are many features available in state-of-the-art ALDs including volume control, wind-

noise management, FM reception, Bluetooth ${ }^{\circledR}$ capabilities, directional microphone, moisture resistance, and ear mold venting ${ }^{7}$ It has been observed that traditional amplification devices does not provide enough aid to ensure natural listening ${ }^{8}$.

The hearing aids available on the market are developed considering the standard ear response, which may not be suitable for all the clients. Many people are dissatisfied with hearing aid even though $10 \%$ of the world population suffers from hearing losses ${ }^{9}$. The development of a client specific hearing aid is not feasible using ASICs. FPAA offers configurable analog subsystem to develop fully configurable HLD as per the patient's audiogram ${ }^{10}$. The average income of users is very less in India and hence the need for developing the low-cost hearing aid technology is consequently growing.

The ANADIGM ${ }^{\mathrm{TM}}$ FPAA synthesis tool makes it fully customizable with ease of analog blocks ${ }^{11}$. This saves the circuit design efforts, cost and time to great extent. The table-1 reveals the degree of hearing losses, which decides level of deafness. This information is very helpful for deciding line of treatment. 


\begin{tabular}{|lc|}
\hline Hearing Losses & Hearing Level $(\mathrm{db})$ \\
\hline Normal & $10-26$ \\
\hline Mild Loss & $27-40$ \\
\hline Moderate Loss & $40-70$ \\
\hline Severe Loss & $70-90$ \\
\hline \hline Profound Loss & Greater than 90 \\
\hline
\end{tabular}

Table 1: Degree of Hearing Losses

The rest of this paper is organized in six sections. In sec 2, system block diagram has been covered followed by goodness factor calculation in sec 3 .FPAA architecture covered in sec 4 followed by results and discussion in sec 5 . Sec 6 covers ear mold fabrication and conclusion in Section 7.

\section{System Block Diagram}

The system block diagram comprises of the major blocks as illustrated in Figure1. Each channel comprises three elements: high pass filter for blocking DC components, a controlled gain stage for to achieve required level of the signal and $2^{\text {nd }}$ order low pass filters to remove the high frequencies due to ambient noise and interferences. Both the filters are based on butter worth functions, which are necessary in the analog stage to have reliable signal information.

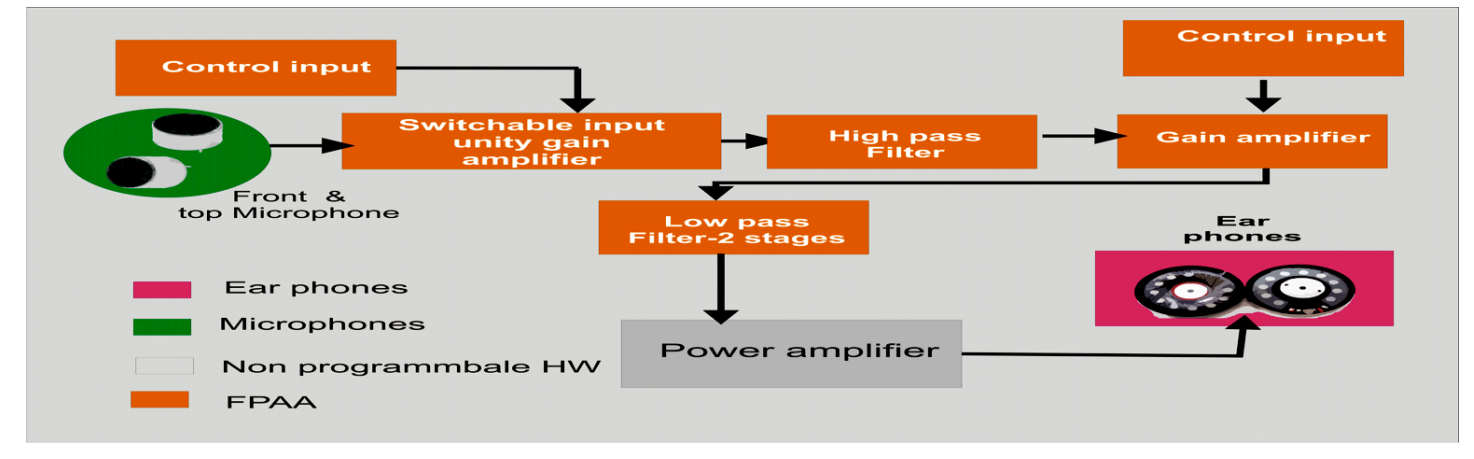

Figure 1: Top Level Block Diagram of HLD.

The controlled gain stage is indispensable since the amplitude of the sound acquired by the microphone is conditioned both by speech and background noise signals. The corner frequencies of the filters are as mentioned in table -2 .

\begin{tabular}{|l|c|c|}
\hline \multicolumn{1}{|c|}{ Filters } & Gain & Corner Frequency \\
\hline High Pass & 1 & $100 \mathrm{HZ}$ \\
\hline Low Pass & 1 & $6 \mathrm{KHZ}$ \\
\hline Low Pass & 1 & $6 \mathrm{KHZ}$ \\
\hline
\end{tabular}

Table 2: Filter Parameters

The selections of cut off frequencies are taken so that less number of filter stages are required to accommodate all blocks on a single device, thus wide marginal value is considered for filter design ${ }^{12}$.

\section{Analog Subsystem Design}

FPAA a customized processor consists of filter, amplifier bank and mixer, In this paper, implementation of the analog system using FPAA platform is illustrated in Figure2. It comprises of analog modules, which processes two input signals fetched from channels and provide a conditioned analog signal. The results obtained for different input signals and estimations of power dissipations and general performance of the system are discussed in result section. The input terminals $\mathrm{n} 1$ and $\mathrm{n} 2$ picks up the signal from front and top microphones, which are operated for half cycle alternatively. The third terminal $\Phi 1$ makes selection of alternate channels or single channel. The $\mathrm{n} 4$ terminal is fed to the input of high pass filter and $\mathrm{n} 3$ controls the gain of the amplifier stage. 


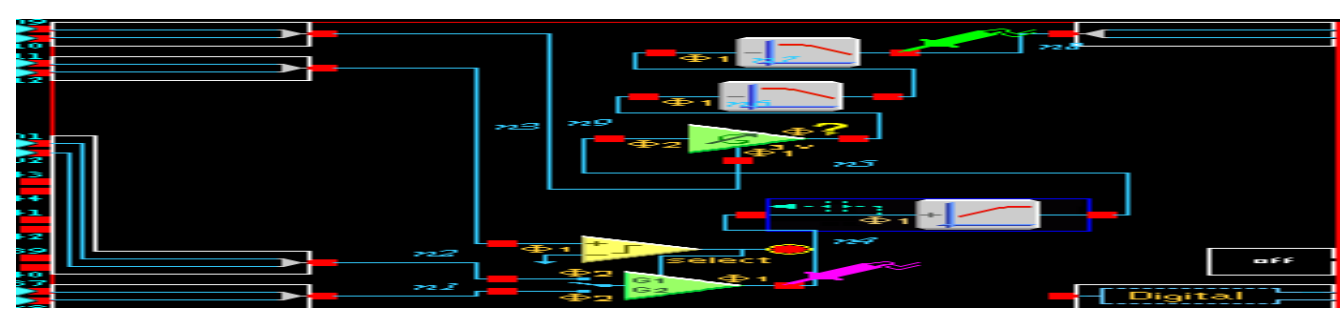

Figure 2 : Analog Subsystem Implementation.

The output of controlled amplifier is passed though two stages of low pass filters with cut off frequencies mentioned in table 2 .

\section{Hearing Threshold Test}

The patient often gives qualitative responses to the questions intended to determine the correctness of a hearing aid. The proposed method of determining the type of aid to fit is by referring the patient's audiogram from a hearing threshold test. The tones can be played directly from the WavGen application software as illustrated in figure 3 . This tool avails the most of the resources of computer and convert computer into audio lab ${ }^{13}$. Hearing loss typically occurs non-uniformly over the audio frequency range.

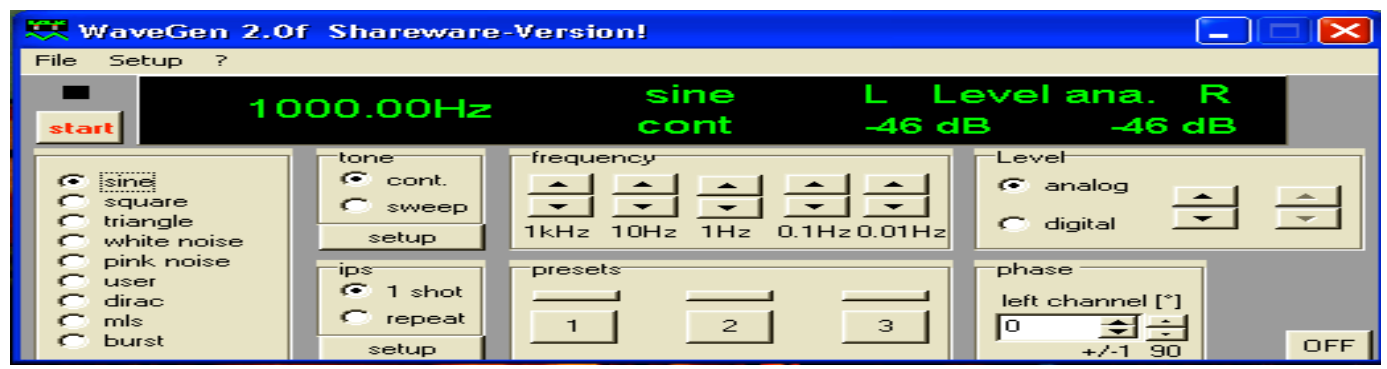

Figure 3: Tone Generator Tool

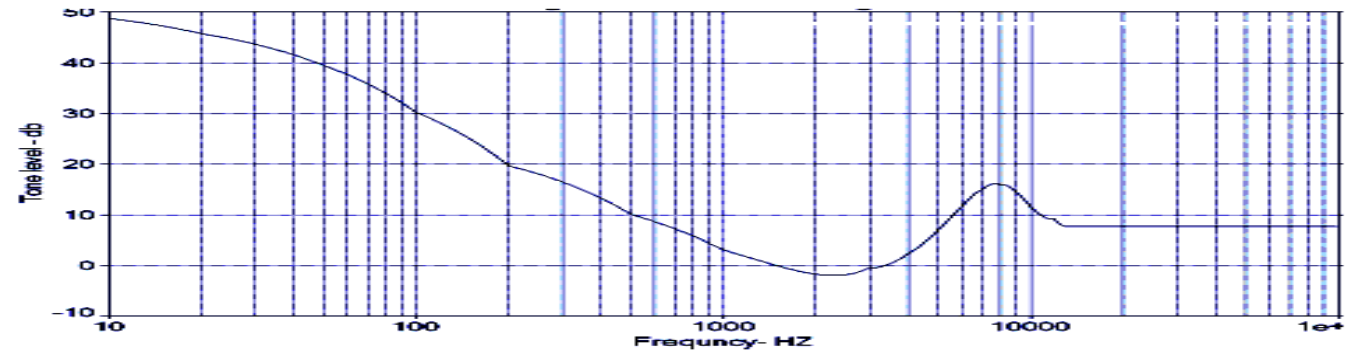

Figure 4: Hearing Threshold Test

In this work, audiogram of hearing impaired persons have been studied with a series of tones and one the samples is presented in figure 4. The volume of each tone (frequency) is adjusted to level that can barely be heard. The values were recorded for each response and the hearing loss w.r.to threshold value was quantified in terms of the gain in $\mathrm{db}^{14}$.

\section{Goodness Factor Calculation}

A goodness factor is considered to be very useful to judge the circuit performance. Considering $\mathrm{H}(\mathrm{j} \omega)$ be the transfer function associated with the circuit on a $\mathrm{dB}$ scale. Assume $\mathrm{E}(\mathrm{j} \omega)$ is the difference between the normal threshold of hearing and client's threshold of hearing (i.e. normal $(\mathrm{dB})-$ client $(\mathrm{dB}))^{15}$. It is the product of the magnitudes of these functions, which equals to 1 for the frequencies.

$$
|H(j \omega)||H(j \omega)|_{d b}=1
$$




$$
\begin{aligned}
& 20 \log (|\mathbf{H}(j w)||\mathbb{E}(j w)|= \\
& 20 \log (|\mathbf{H}(j w)|)+20 \log (|\mathbb{E}(j w)|=0
\end{aligned}
$$

It has been observed that it is very difficult task to test the ear at all frequencies. A set of test points to use complete audio range has been determined. Assume that $\mathrm{N}$ samples were used in testing to predict hearing loss, the bias error for the ALD and ear is given by the average error in terms of $\mathrm{dB}$

$\mu_{\mathrm{e}}=$ bias_error $=$

$$
\frac{1}{N_{i}} \sum_{=1}^{N} 20 \log (|\hat{H}(j 2 \pi f i)|)+20 \log (|\hat{E}(j 2 \pi f i)|)
$$

The variation about the normal criterion is expressed as:

$$
\begin{aligned}
& \sigma e^{2}=\text { variance_error }= \\
& \frac{1}{\mathrm{~N}} \sum_{\mathrm{i}=1}^{\mathrm{N}}[20 \log (\hat{\mathrm{H}}(\mathrm{j} 2 \pi \mathrm{fi}) \mid)+20 \log (\hat{\mathrm{E}}(\mathrm{j} 2 \pi \mathrm{fi}) \mid)-\mu \mathrm{e}]^{2}
\end{aligned}
$$

\section{Analog Subsystem}

FPAA offers feasibility of alteration of filter parameters. This technology offers an interesting tradeoff between development- configuration time and its performance. This rapidity is appreciable in contrast with other devices i.e. switched capacitor filters etc. Thus this feature forces us to think innovative about the selection of FPAA device. There are many variants of FPAA from Motorola, Lattice, Cypress, ANADIGM ${ }^{\mathrm{TM}}$, Zetec etc.

\section{Device Configuration}

For the implementation of the analog system, AN221E04 FPAA from ANADIGM ${ }^{\mathrm{TM}}$ was configured. The FPAA comprises four input/output blocks, which can be utilised as differential or single ended interfaces, and two output blocks, which also can be programmed as low pass filters. The configurable analog input signals are connected to a bank of analog switches, connected again to eight programmable capacitors, which can take a value between 0 and 255 units of capacitance. The bank of capacitors is connected to a second bank of analog switches, to establish the circuit topology. Since the output of this second bank is connected to three operational amplifiers, one of them is working as analog comparator. The output of these devices are connected both back to the first bank of switches and to a multiplexer which drive them to the $\mathrm{CAB}$ (configurable analog block) output. Each CAB has a LUT (look up table) interface to connect it to the main FPAA LUT.

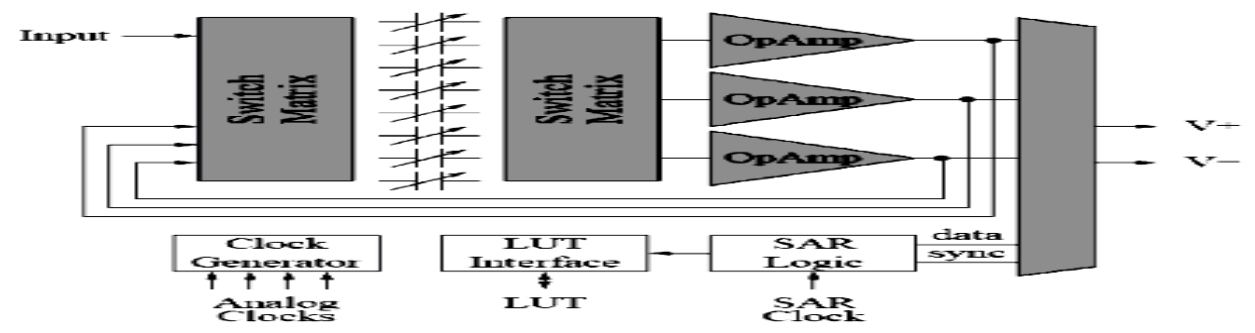

Figure 5: Configurable Analog Block ${ }^{16,17}$

It has a clock generator connected to the four clock signals coming from the main device clock circuitry. SAR (Successive approximation register logic) is considered as very important block needed to synthesize some functions like the analog multiplication.

\section{Results and Discussion}


MATLAB program for generating user defined single or composite tones in wave audio format was developed.

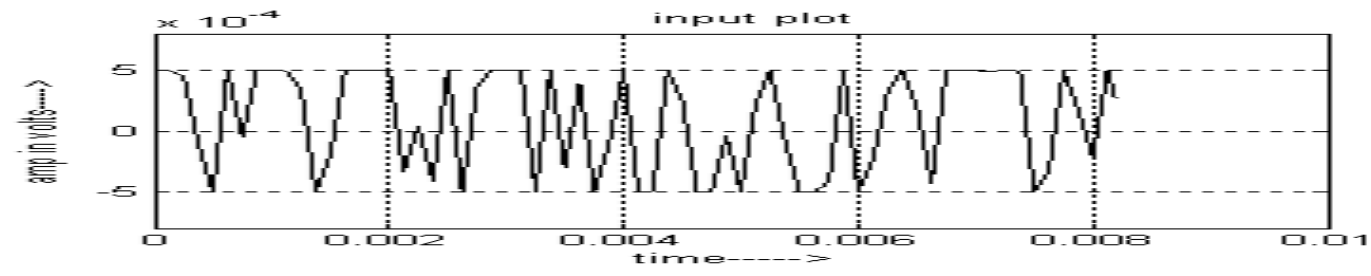

Figure 6: Composite Tone

The signal without and with noise are illustrated in figure 6 and figure 7. The frequency plot reveals about the various components of tone as depicted in figure 8 , which is applied to the analog subsystem as an input. The output of an analog system has been plotted in time domain and saved as Microsoft EXCEL ${ }^{\mathrm{TM}}$ file with .csv extension.

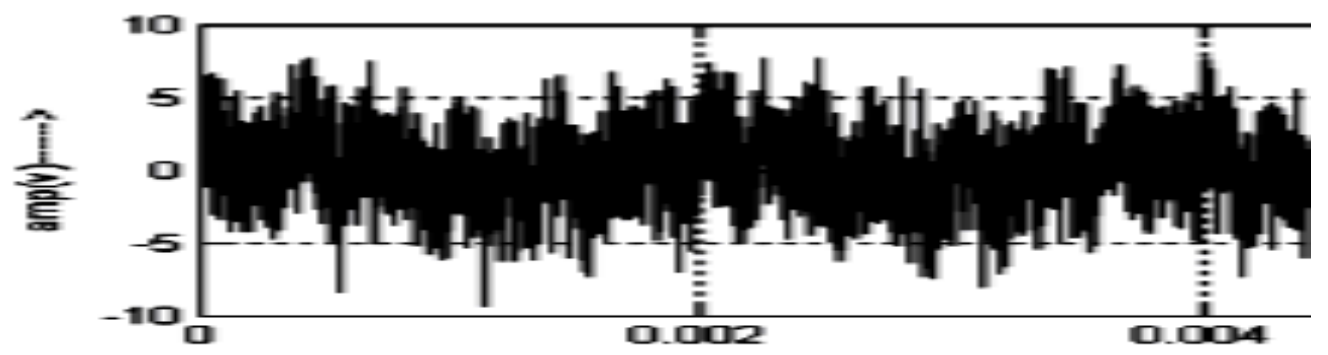

Figure 7: Composite Tone with Noise

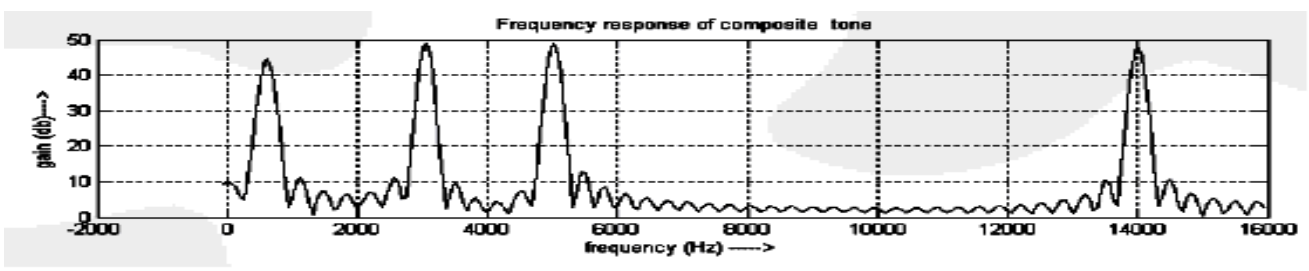

Figure 8: Frequency Plot of Composite Tone

For testing purpose, only one channel was considered. The channel selection can be done by controlling $\Phi_{1}$ terminal.

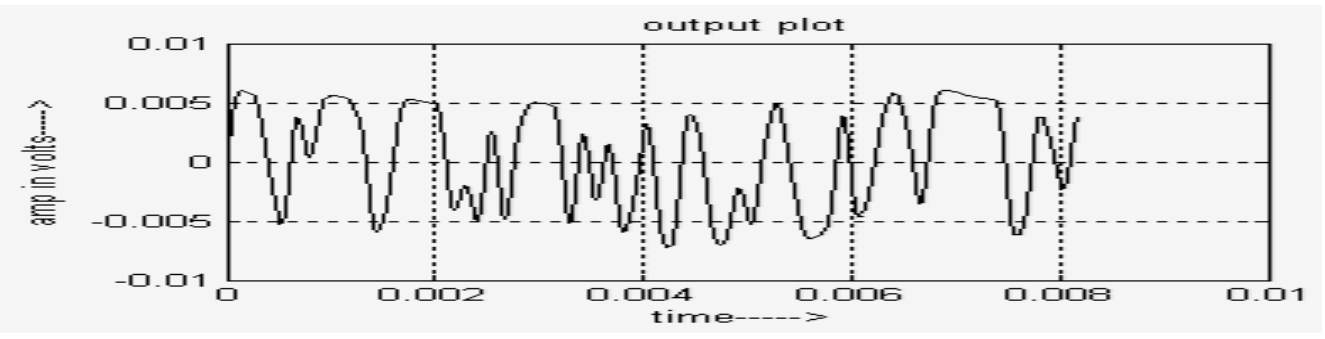

Figure 9: Output of FPAA Based Subsystem

MATLAB program ${ }^{18}$ was used to import the excel files. The output gained from the system is filtered and amplified as shown in figure 9. The estimated power dissipation, capacitors, operational amplifiers, comparators and successive registers in each configurable analog block is evaluated. It can be noted that the use of the FPAA is quite intensive. The frequency response of output signal speaks about the system performance w.r.t. frequency components as illustrated in figure 10 . 


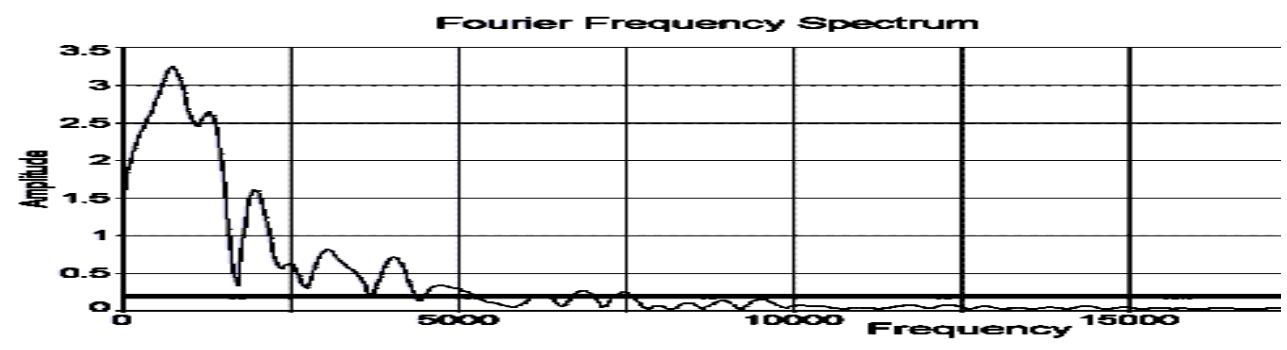

Figure 10: Frequency Spectrum of an Output.

The following statistics estimate various resources of FPAA and power dissipation of the analog system as shown in table-3.

\begin{tabular}{|c|c|c|c|c|}
\hline CAB & CAPS & OP-AMPS & COMPS & SAR \\
\hline 1 & 6 & 2 & 1 & 0 \\
\hline 2 & 7 & 2 & 1 & 1 \\
\hline 3 & 3 & 1 & 0 & 0 \\
\hline 4 & 0 & 0 & 0 & 0 \\
\hline TOTAL & 16 & 5 & 2 & 1 \\
\hline$\%$ utilization & 50 & 62.5 & 50 & 25 \\
\hline \multicolumn{5}{|c}{ Power Consumption in m: $295 \pm 89$} \\
\hline
\end{tabular}

Table 3: FPAA Resource Utilization

\section{Fabrication of Ear Fitment and System Packaging}

Ear molds are the plastic part, which connect ALD and the ear canal and literally place the sound in your ear. Custom ear molds are a great way to protect your hearing from loud sounds at work. It is recommended to make a cast of an ear to ensure right fitment. In this work, we proposed ear mold made up of biocompatible material along with fabrication flow. There are many research literatures in which acrylic materials used but it is not truly flexible.

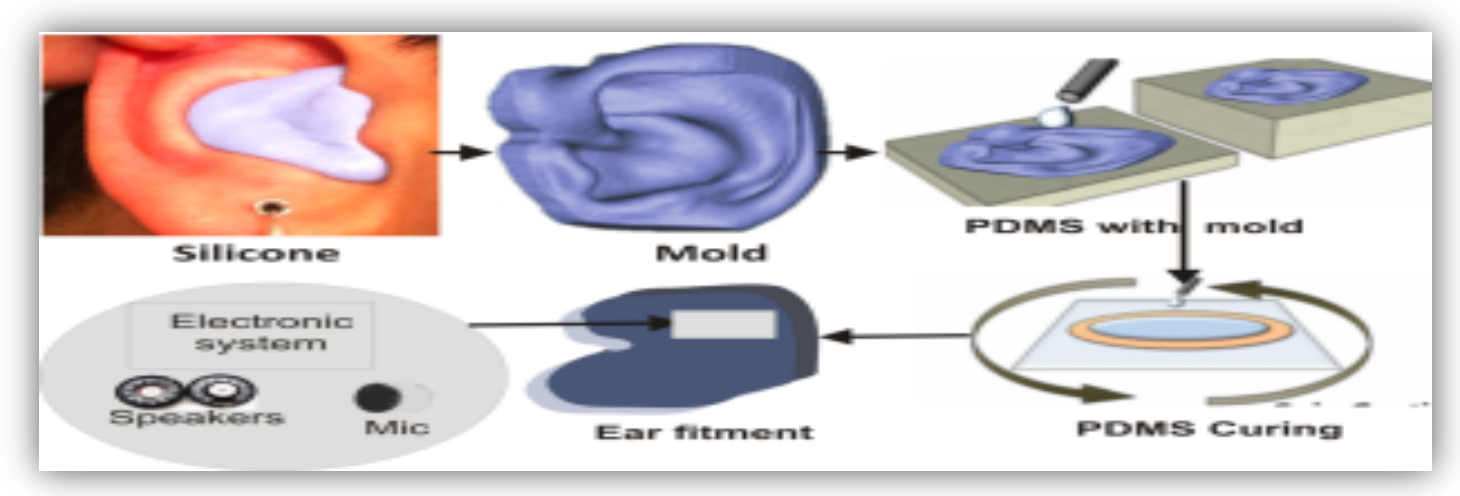

Figure 11: Ear Mold and Fitment Fabrication

Polyvinyl chloride (PVC), is the most preferred material considered as flexible. Furthermore, vinyl ear molds are not generally recommended for use with patients who have allergy concerns. It is proposed to use silicone material for an ear mold.

PDMS elastomer is a widely used bio-material for ear fitment because of its biocompatibility, ease of fabrication by spin-coating or Molding the liquid ${ }^{19,20,21,22}$. The main advantage of polymer lies in their low elastic modulus, which improves flexibility and comfort. PDMS (Sylgard 184 kit from Dow Corning) and curing agent were mixed thoroughly in weight ratio of 10:1 for fabrication of ear fitment. FPAA based system, microphones and earpieces can be embedded in PDMS polymer as illustrated in figure11.

\section{Conclusion}


In this paper, the rapid implementation of an analog subsystem that has two input channels for front and top microphones in multiplexed mode has been presented. The testing reports reveal the practical feasibility required to satisfy the client's requirement. The customized design using FPAA makes alteration easier and quicker. In addition to this, fabrication process of ear fitment made up of polymer is also discussed in this work. In future, this work would be continued to embed digital processing module, bluetooth ${ }^{\mathrm{TM}}$ connectivity and inbuilt memory in the same capsule.

\section{References}

1. Y. Lin, Y. Lai, H. Chang, Y. Tsao, Y. Chang and R. Y. Chang, "SmartHear: A Smartphone-Based Remote Microphone Hearing Assistive System Using Wireless Technologies," in IEEE Systems Journal, vol. 12, no. 1, pp. 20-29, March 2018.

2. M. S. Mahamud and M. S. R. Zishan, "Watch IT: An assistive device for deaf and hearing impaired," 2017 4th International Conference on Advances in Electrical Engineering (ICAEE), Dhaka, 2017, pp. 556-560.

3. https://www.floridahearingsociety.com/hearing-technology

4. Flaubert JL, Spicer CM, Jette AM, editors. The Promise of Assistive Technology to Enhance Activity and Work Participation. Washington (DC): National Academies Press (US); 2017 May 9. 5.

5. S. Nordholm, W. Kellermann, S. Doclo, V. Valimaki, S. Makino and J. Hershey, "Signal Processing Techniques for Assisted Listening [From the Guest Editors]," in IEEE Signal Processing Magazine, vol. 32, no. 2, pp. 16-17, March 2015.

6. Suresh S. Balpande, et.al., "Modeling of Cantilever Based Power Harvester as an Innovative Power Source for RFID Tag", IEEE International Conference on Emerging Trends in Engineering \& Technology, pp. 13-18, 2009. DOI: 10.1109/ICETET.2009.152.

7. John DiCristina, Introduction to Hearing Aids and Important Design Considerations, whitepaper, https://www.maximintegrated.com/en/app-notes/index. mvp/id/4691

8. Alice E. Holmes, Beyond the hearing aid: Assistive listening devices, The Journal of the Acoustical Society of America 113, 2221 (2003).

9. Othman khalifa, M.H. Makhtar \& M.S. Baharom 'Hearing aid system for impaired peoples", International journal of computing and information sciences, Vol 2 no.1 -April 2004 ,pp 23-26.

10. T. B. Deng., "Three-channel variable filter-bank for digital hearing aids" IEEE Transaction on Signal Processing, 4(2),2010, pp. $181-196$.

11. Gines Domenech-Asensi, Juan Martinez Alajarin, Ramon Ruiz \& Merino, ,'Synthesis on FPAA Of A Smart sthetoscope Analog Subsystem", IEEE explore, 2006.

12. D. George Gata, Walter Sjursen \& James R. Hochschild ," A 1.1-V 270- A Mixed-Signal Hearing Aid Chip", IEEE Journal of solid-state Circuits, vol. 37, no. 12, December 2002.

13. WaveGen 2.0f tone generator tool help file.

14. R. E. E. Robinson, "A DSP hearing aid simulator and screening test", Speech Hearing and Language Research Centre, Macquarie University.

15. "Custom Design of a High-Fidelity Hearing Aid", Web source: http://www.engr.uky.edu/ donohue/audio/ fsear.html.

16. Andrzej Malcher And Piotr Falkowski, Analog Reconfigurable Circuits, Intl Journal of Electronics And Telecommunications, 2014, Vol. 60, No. 1, Pp. 15-26, DOI: 10.2478/Eletel-2014-0002.

17. Cheng Xiaoyan et al 2014 J. Semicond. 35105011

18. Doug hull, Building a GUI to read an excel file in guide of MATLAB,. http//blogs.mathworks. com/pick .

19. Suresh S. Balpande, Rajesh S. Pande, Rajendra M. Patrikar, "Design and low cost fabrication of green vibration energy harvester", Sensors and Actuators A: Physical, Volume 251, 1 November 2016, Pages 134-141, ISSN 09244247.

20. Balpande, S.S.; Bhaiyya, M.L.; Pande, R.S.: "Low-cost fabrication of polymer substrate-based piezoelectric microgenerator with PPE, IDE and ME", Electronics Letters, 2017, 53, (5), p. 341-343

21. Mayuri D. Dhone, Poonam G. Gawatre, Suresh S. Balpande, "Frequency band widening technique for cantilever-based vibration energy harvesters through dynamics of fluid motion" ,Materials Science for Energy Technologies, Volume 1, Issue 1,2018,Pages 84-90

22. Suresh S. Balpande, R.S. Pande, Design and fabrication of non silicon substrate based MEMS energy harvester for arbitrary surface applications, AIP Conf. Proc. 1724 (2016) 020099, pp 020099-1-7. 